Kragujevac Journal of Mathematics

Volume 40(2) (2016), Pages 192-193.

\title{
A REMARK ON THE PAPER "ON WEAKLY SYMMETRIC SPACETIMES" (KRAGUJEVAC J. MATH. 36(2) (2012), 299-308)
}

\author{
KANAK KANTI BAISHYA
}

ABSTRACT. The object of the present paper is to rectify the example in Section 5 and nullify the Theorem 5.1.

\section{REMARK}

In [1], the authors have stated that:

(a) [1, Theorem 5.1, p. 306] "Let us consider a Lorentzian metric $g$ on $\mathbb{R}^{4}$ by

$$
d s^{2}=g_{i j} d x^{i} d x^{j}=x^{2}\left[\left(d x^{1}\right)^{2}+\left(d x^{2}\right)^{2}+\left(d x^{3}\right)^{2}\right]-\left(d x^{4}\right)^{2},
$$

where $i, j=1,2,3,4$. Then $\left(\mathbb{R}^{4}, g\right)$ is a weakly symmetric spacetime whose scalar curvature is non-zero and non-constant".

But statement (a) is found to be false. Let us recall the definition of a weakly symmetric manifold.

A non-flat Riemannian manifold is called a weakly symmetric manifold if it realizes the relation $(1.2)$ of $[1$, p. 300]. The local expression of the relation $(1.2)$ of $[1$, p. 300], is

$$
R_{h i j k, l}=A_{l} R_{h i j k}+D_{h} R_{l i j k}+D_{i} R_{h l j k}+D_{j} R_{h i l k}+D_{k} R_{h i j l}
$$

where $A_{l}$ and $D_{l}$ are two non-zero co-vectors and comma followed by indices denotes the covariant differentiation with respect to the metric tensor $g$. An $n$-dimensional manifold of this kind is denoted by $W S_{n}$.

Key words and phrases. Weakly symmetric spacetimes. 2010 Mathematics Subject Classification. 53C15, 53C25.

Received: February 16, 2016.

Accepted: May 30, 2016. 
The covariant derivative of the Riemann curvature tensor $R_{h i j k}$ is defined as $[2$, p. 85]

$$
R_{h i j k, l}=\frac{\partial R_{h i j k}}{\partial x^{l}}-\Gamma_{h l}^{a} R_{a i j k}-\Gamma_{i l}^{a} R_{h a j k}-\Gamma_{j l}^{a} R_{h i a k}-\Gamma_{k l}^{a} R_{h i j a}
$$

The only non-vanishing components of the Christoffel symbols and the Riemann curvature tensor are [1, p. 305-306]

$$
\begin{aligned}
\Gamma_{12}^{1} & =\Gamma_{23}^{3}=\Gamma_{22}^{2}=-\Gamma_{11}^{2}=-\Gamma_{33}^{2}=\frac{1}{2 x^{2}}, \\
R_{1221} & =R_{2332}=-\frac{1}{2 x^{2}}, R_{1331}=\frac{1}{4 x^{2}},
\end{aligned}
$$

obtained by the symmetry and skew-symmetry properties of the above mentioned components. Now making use of the definition of the covariant derivative, we have

$$
\begin{aligned}
R_{2331,1} & =\frac{\partial R_{2331}}{\partial x^{1}}-\Gamma_{12}^{a} R_{a 331}-\Gamma_{13}^{a} R_{2 a 31}-\Gamma_{13}^{a} R_{23 a 1}-\Gamma_{11}^{a} R_{233 a} \\
& =-\Gamma_{12}^{1} R_{1331}-\Gamma_{11}^{2} R_{2332}=-\frac{3}{8} \cdot \frac{1}{\left(x^{2}\right)^{2}} .
\end{aligned}
$$

In a similar manner, we can find $R_{1221,2}=\frac{3}{2} \cdot \frac{1}{\left(x^{2}\right)^{2}}$ and $R_{1331,2}=-\frac{3}{4} \cdot \frac{1}{\left(x^{2}\right)^{2}}$. Now, by virtue of (1.1) and $R_{2331,1}$, we have

$$
\begin{aligned}
R_{2331,1} & =A_{1} R_{2331}+D_{2} R_{1331}+D_{3} R_{2131}+D_{3} R_{2311}+D_{1} R_{2331} \\
& \Rightarrow D_{2}=-\frac{3}{2} \cdot \frac{1}{x^{2}}, \text { as } R_{2331}=0=R_{2131}=R_{2311} .
\end{aligned}
$$

Analogously, from (1.1), $R_{1221,2}$ and $R_{1331,2}$ we can easily bring out $A_{2}=-\frac{3}{x^{2}}$ and $D_{2}=\frac{1}{x^{2}} \neq-\frac{3}{2} \cdot \frac{1}{x^{2}}$.

Consequently, the spacetime $\left(\mathbb{R}^{4}, g\right)$ under considered metric $g$ can not be a $W S_{4}$. This completes the proof that statement (a) is false.

Acknowledgements. The author would like to express his sincere thanks to the referees for their valuable suggestions to improve this manuscript.

\section{REFERENCES}

[1] U. C. De and S. Mallick, On weakly symmetric spacetimes, Kragujevac J. Math. 36 (2) (2012), 299-308.

[2] I. S. Sokolnikoff, Tensor Analysis-Theory and Applications, John Wiley \& Sons, New York, 1951.

Department of Mathematics,

Kurseong College, Dowhill Road, Kurseong,

DARJEeling-734203, West Bengal, IndiA

E-mail address: kanakkanti.kc@gmail.com 